(C) 2014 IEEE. Personal use of this material is permitted. Permission from IEEE must be obtained for all other uses, in any current or future media, including reprinting/republishing this material for advertising or promotional purposes, creating new collective works, for resale or redistribution to servers or lists, or reuse of any copyrighted component of this work in other works. 


\title{
Path-loss and car-body-effect characterization for smart tires communications at UWB and ISM bands
}

\author{
S. Ghamari, G. Tasselli, Y. Guo, C. Robert, C. Botteron and P.-A. Farine \\ Ecole Polytechnique Fédérale de Lausanne (EPFL) / Electronics and Signal Processing Laboratory, \\ Neuchâtel, Switzerland. \\ Contact: saeed.ghamari@epfl.ch
}

\begin{abstract}
Next generation of smart tires will improve the safety and stability of the vehicle by monitoring road parameters, such as pavement and rolling conditions, with sensors placed on tire surface. Between the tread and the metal belt of the tire there are only few millimeters of rubber, therefore high miniaturization is necessary to withstand at the extremely high accelerations and to fit in this very limited room. Such kind of miniaturization poses huge limitations on antenna efficiency and available power likely provided by energy scavengers. Therefore, the optimization of the wireless power budget is crucial and it passes through the study of the communication channel. This paper presents a characterization setup that measures the pathloss from the tire surface to the inside of the car where the standard RF receivers, such as RKE at $434 \mathrm{MHz}$ and Bluetooth at $2.4 \mathrm{GHz}$, are usually located. A calibration procedure has been employed in order to de-embed car-body-effects from the overal path-loss. Moreover, a 4.1 GHz UWB radio has also been considered in this study motivated by the extremely low-power consumption reached by UWB transmitters. The measurement results reflect a complex propagation environment where the car body attenuation plays a marginal role. Instead, the antenna pattern and the reflections from the environment cause the largest attenuation. Moreover, the observed link budget margin is larger and more suitable for implementation within the ISM bands than for the UWB band.
\end{abstract}

Keywords- Smart tires; Path-loss characterization; Car-body effect; Vehicle attenuation; RKE; UWB;2.4GHz ISM, 434MHz ISM.

\section{INTRODUCTION}

The research activity into smart tires, also known as intelligent tires, has been triggered by the United States transportation recall enhancement, accountability, and documentation (TREAD) act, in 2001, which demands installation of tire pressure monitoring system (TMPS) within the tire for the new cars [1]. A similar legislation has been adopted in South Korea since 2013 and also in Europe by 2014.

So far, attention of designers has been focused on sensors attached to the inner part of the tire or clamped on the rim, below the metal belt which forms the tire's frame. The metal belt consists of a grid of steel cords that cannot be holed, for safety and reliability reasons, to pass wires for the interconnections between an outer sensor, eventually embedded in the tread, and the inner circuitries. On the other hand, monitoring parameters such as pavement and rolling conditions are expected to improve the reliability of the tires and tire control systems such as anti-lock braking systems (ABS) and vehicle stability systems. In this case, sensors placed outside the tire's metal belt must be autonomous for the power supply and wirelessly connected to the vehicle's electronic box [2]. Moreover, the selected RF interface with the car should be a standard interface not to be dependent of a given tire manufacturer, and if possible, should be based on some already available RF interfaces of the car, such as the remote keyless entry (RKE) or the Bluetooth (BT), to yield a cost-effective solution for the smart tires. The development of sensors to embed outside the metal belt of the tire is therefore a challenging task because of the required miniaturization necessary to withstand at the extremely high acceleration and to fit in the limited room between the tread and the metal belt. Such kind of miniaturization poses huge limitations on antenna efficiency and available power likely provided by energy scavengers[3]; both factors negatively affect the wireless communication capabilities of the sensor. The optimization of the wireless power budget is therefore crucial and it passes through the study of the communication channel.

Recently, some channel characterizations at industrial, scientific and medical (ISM) bands (434 MHz and $2.4 \mathrm{GHz}$ ) and UWB band (3.1-10.6 GHz) have been reported. However, they are focused on the TMPS application where the sensor is located below the metal belt and the receiver is located in the car's central console [4]-[6]. These characterizations show the communication feasibility and the absolute power level at the receiver side, but do not provide a breakdown of the path-loss (PL) contributions.

In this paper, we use a characterization setup to measure the PL from the tire surface to the receiver end, excluding the shielding effect of the metal belt which can be added by studying solely the wheel when required. The receiver locations have been chosen between the actual locations of the RKE and the BT receiver because they are the standard RF access points of the cars. The investigated frequencies are the $434 \mathrm{MHz}$ used by the European RKE standard, the $2.4 \mathrm{GHz}$ BT low-energy band and 4.1 GHz selected within the 3.1-10.6 $\mathrm{GHz}$ UWB band. Finally, a calibration to evaluate the carbody-effect and a break-down of the PL contributions are provided as they are important for power budget optimizations. 


\section{MEASUREMENT SETUP}

The measurement setup for a static characterization of the PL has been defined in a way to reproduce, as close as possible, the real operating circumstances of a car. A parking area has been chosen because it has the same elements of a road such as asphalt paving and reflecting elements within few meters of distance. The main characteristics are summarized in Table I. Vehicle dimensions and positions of transmitters and receivers are shown in Fig. 1 and Fig. 2. Note that the origin of the coordinate system is the center of the front-left wheel.

TABLE I. MAIN CHARACTERISTICS OF THE MEASUREMENT SETUP

\begin{tabular}{|c|c|}
\hline Parameter & Description \\
\hline Car model & $\begin{array}{c}\text { Seat Altea XL-normal windows, } \\
\text { closed windows and doors. }\end{array}$ \\
\hline Wheelbase & $258 \mathrm{~cm}$ \\
\hline Axle track & $154 \mathrm{~cm}$ \\
\hline Sumber of passengers on board & 1 \\
\hline Environment & Asphalt \\
\hline Weather condition & Parking area \\
\hline
\end{tabular}

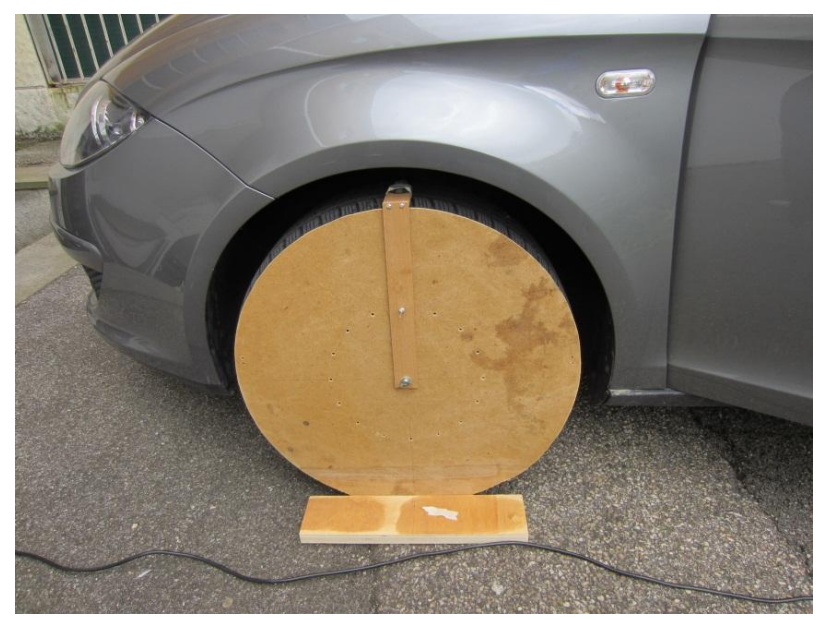

Fig. 1. Transmitter located on the wheel $[0,0,30] \mathrm{cm}$. Origin of the coordinate system is the center of the front-left wheel

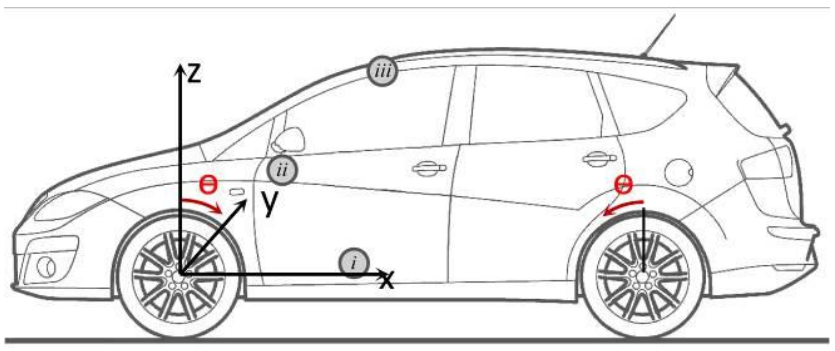

Fig. 2. Receiver position inside the car: (i)- RX Antenna in the door pocket [105, 0, 30] cm. (ii)- RX Antenna on the central console [95, 85, 70] cm, (iii)RX Antenna on the ceiling light $[95,85,100] \mathrm{cm}$.

The transmitter is placed on the tire by means of a wooden stand that allows its rotation around the tire with $22.5^{\circ}$ of angle resolution (see Fig. 1). This enables the simulation of the rotation of the wheel during vehicle movement. The transmitting antenna can be placed as close as $5 \mathrm{~mm}$ to the tire surface. The receiving antenna has been positioned in three different positions (see Fig. 2), considering the actual locations of the RKE receiver in most of the cars on the market. These positions are: the pocket in the driver's door (setup i), the central console (setup ii) and the ceiling light just over the internal mirror (setup iii). A person is seated on the driver's place to mimic real operating conditions.

\section{CHANNEL CHARACTERIZATION WITHIN ISM BANDS.}

Wave propagation around a car in a realistic environment, even in static conditions, is quite complex to model. It cannot be approximated with simple models such as free-space path loss (FSPL) or log-distance because of the complex shape of the car, its heterogeneous materials and also the reflections of the road and the environment. Moreover, the small room available around the wheels and inside the car imposes the use of small antennas placed very close to reflecting objects. As a result, the antenna radiation patterns are far from the isotropic case. Indeed, they show some nulls which are visible in the measurement results. By definition, PL is estimated by equation (1):

$$
P_{L}[d B]=10 \log _{10}\left(\frac{P_{T X}}{P_{R X}}\right),
$$

where $\mathrm{P}_{\mathrm{RX}}$ and $\mathrm{P}_{\mathrm{TX}}$ are the received and transmitted powers, respectively. Since $P_{R X}$ is an absolute value, it embeds many factors that are not related to the propagation channel such as cable losses, antenna gain and directivity, instruments inaccuracy, etc. Measuring $\mathrm{P}_{\mathrm{RX}}$ without the car is a useful step because it helps to better understand the aforementioned effects on the PL. Therefore, a calibration step has been employed in our measurement setup. This step has a twofold purpose: one is to separate the car effects from the environment effects in order to estimate the car body attenuation, and another is to study the antennas and the environment effects (i.e. interactions with the wooden holders and the ground effects) in absence of the car. The calibration can be done by measuring the received power for each position of the transmitter in each considered setup with and without the vehicle while keeping the coordinates of the transmitter and the receiver fix for each case. The difference between these two received powers is the vehicle body attenuation which can be expressed in $\mathrm{dB}$ by (2):

$$
L_{C B}[d B]=10 \log _{10}\left[\frac{P_{R X \text {-without car }}}{P_{R X \text {-withcar }}}\right] .
$$

\section{A. Calibration of the measurement setup at $434 \mathrm{MHz}$}

The transmitter consists of the "4010-KFOB-434" board of the Si4010 development kit housed in a plastic $43 \times 27 \mathrm{~mm}^{2}$ [7]. The transmitted power is set to $10 \mathrm{dBm}$ and the radiated power by the antenna is given in Table II; the latter shows $20 \mathrm{~dB}$ of variation depending on the orientation of the receiving antenna.

In order to measure the received power we used a spectrum analyzer (FSP315 by R\&S) and a helical antenna with $0 \mathrm{dBi}$ gain and isotropical radiation on its horizontal plane [8]. The spectrum analyzer has been placed in the driver's footwell. A schematic representation of the calibration setup, for the case 
of transmission between the $0^{\circ}$ wheel-rotation of the front-left wheel and the door pocket (i) is shown in Fig. 3. Since we have considered three receiving antenna positions (i-iii, see Fig. 2), the calibration procedure has been repeated three times.

TABLE II. MAXIMUM OF THE EIRP OF THE 4010-KFOB-434 BOARD FOR 10dBm OF GENERATED POWER [7].

\begin{tabular}{|c|c|c|c|}
\hline \multirow{3}{*}{$\begin{array}{l}\text { Receiving } \\
\text { antenna } \\
\text { Polarization }\end{array}$} & \multicolumn{3}{|c|}{ EIRP [dBm] } \\
\hline & & Plane & \\
\hline & $X Y$ & $Z Y$ & $Z X$ \\
\hline H & -11.39 & -19.37 & -19.83 \\
\hline V & -30.61 & -17.67 & -18.42 \\
\hline $\begin{array}{c}\text { TX board } \\
4010-\mathrm{KFOB}\end{array}$ & & & $\begin{array}{c}\text { Helix } \\
\text { Antenna } \\
\text { TI.10.0112 }\end{array}$ \\
\hline
\end{tabular}

Fig. 3. Schematic representation of the calibration setup (434MHz, setup i).

Fig. 4 shows the comparison between the theoretical FSPL and the measured PL for the calibration setup shown in Fig. 3, in which the losses consisting in cables losses and antennas losses are estimated to $25 \mathrm{~dB}$. It can be noted that the theoretical FSPL is a poor approximation even for the calibration setup that does not include car-body-effects.

\section{B. Calibration of the measurement setup at $2.4 \mathrm{GHz}$}

The calibration procedure is the same as for the case of $434 \mathrm{MHz}$ except that it has been done only for setup (ii). For convenience a ZigBee nodes by NXP, named JN5148-001M00 [9], has been used. Indeed BT shares the same frequency band. The device is characterized by a sensitivity of $-95 \mathrm{dBm}$ when working at $250 \mathrm{Kbit} / \mathrm{s}$. The printed antenna has a typical loss of about $4 \mathrm{~dB}$, therefore the maximum Effective Isotropic Radiated Power (EIRP) is $-1.5 \mathrm{dBm}$. One node has been programmed as coordinator and the other as end-device. The coordinator establishes the network and collects the received signal strength indicator (RSSI) values. Every two seconds four packets are sent and a RSSI value is sent on the USB port and shown on the laptop screen. The printed data have been saved on files to be analyzed in post processing.

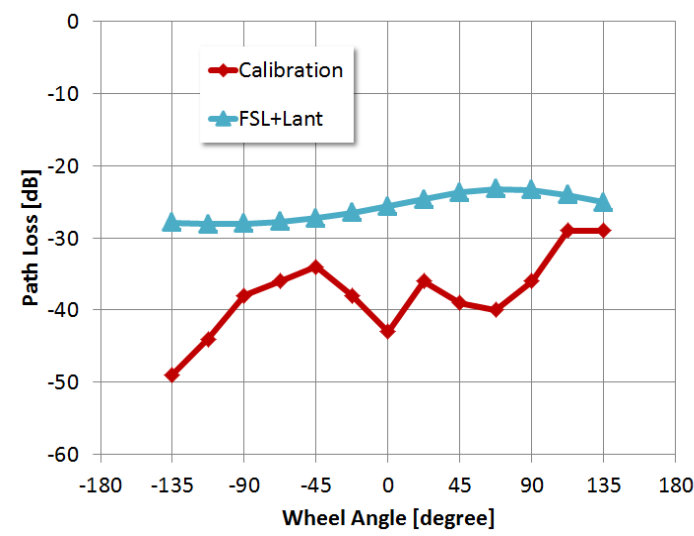

Fig. 4. Comparison between FSPL model and PL measured for the calibration setup (i). Lant are the antenna and cable losses $\sim 25 \mathrm{~dB}$.

\section{ISM BAND MEASUREMENT RESULTS}

\section{A. Measurement Results at $434 \mathrm{MHz}$}

The PL characterization at $434 \mathrm{MHz}$ has been done for the three different receiver setups depicted in Fig. 2. In Fig. 5, the four lines are related to the wheels of the car and the acronym meanings are: $\mathrm{FL}=$ front-left, $\mathrm{FR}=$ front-right, $\mathrm{RL}=$ rear-left and $\mathrm{RR}=$ rear-right. The received power in the case of setup (i) is plotted versus wheel angle which is clockwise for FL and RR wheels and counterclockwise for the others. The typical receiver sensitivity $(-110 \mathrm{dBm})$ is marked with a dashed-line in the figure to show the link margin. The power of the transmitter is $10 \mathrm{dBm}$ and the estimated losses are $25 \mathrm{~dB}$ for all the cases (due to the $-22 \mathrm{~dB}$ TX antenna gain (see Table II) and $3 \mathrm{~dB}$ cable losses).Therefore, the PL can be read by adding $15 \mathrm{~dB}$ to the power level of Fig. 5. To provide more insight, the extreme power limits and corresponding PLs for the different setups are summarized in Table III.

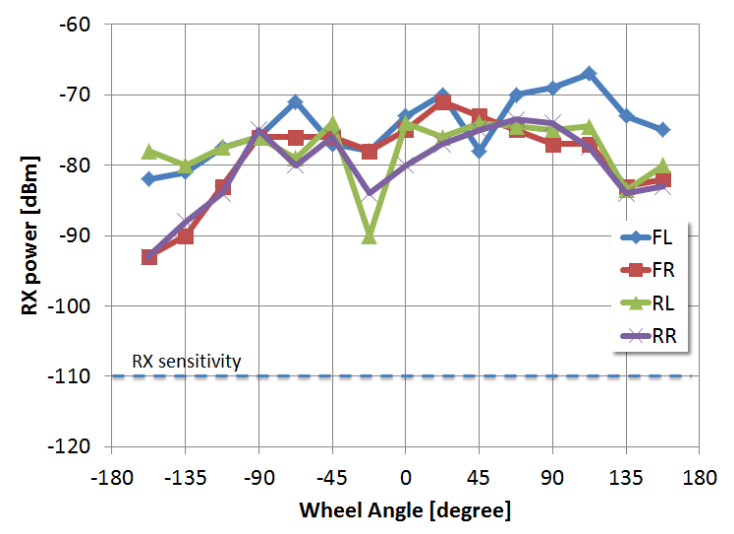

Fig. 5. Received power at $434 \mathrm{MHz}$ for the case of setup (i) versus wheel angle. $\mathrm{P}_{\mathrm{TX}}=10 \mathrm{dBm}$ and estimated antennas and cables losses are $25 \mathrm{~dB}$.

Fig. 5 is related to setup (i) and shows a $P_{R X}$ variation of $26 \mathrm{~dB}$. The minimum peaks are not always correlated to transmitter bottom position. Indeed, the transmitted EIRP changes between $-12 \mathrm{dBm}$ to $-30 \mathrm{dBm}$ depending on its orientation, therefore $18 \mathrm{~dB}$ of variation could be attributed to the rotation of the transmitting antenna. The remaining part is due to the other factors such as soil proximity, reflections and receiving antenna radiation pattern. Restricting the operating angle to the top quarter of the wheel the power variation range is reduced by 6 , 10 and $6 \mathrm{~dB}$ for setups (i.ii. and iii), respectively. On the other hand, the minimum power increases only by 3,5 and $6 \mathrm{~dB}$ for the same cases. This makes the top quarter of the wheel as a better position for the transmitter to function. However, this restriction is applicable only if the packet takes less than the usable angle to be transmitted. Since the transmission is not synchronized with the wheel angle the probability to have a successful transmission increases as the packet length decreases, i.e. when data rate increases. It can be observed that about $7 \mathrm{~dB}$ of variation in setup (i) is due to the negative peak happening for the RL wheel at $-22.5^{\circ}$. It could be caused by antenna pattern nulls as well as multipath effects. 
TABLE III. MEASURED PL AND RX POWER OVER DIFFERENT ANGLE RANGES AT 434MHZ.

\begin{tabular}{|c|c|c|c|c|}
\hline & & $\begin{array}{c}\text { Full } \\
\text { angle }\end{array}$ & $\begin{array}{c}\text { Top half } \\
\left(-90^{\circ} \text { to } 90^{\circ}\right)\end{array}$ & $\begin{array}{l}\text { Top quarter } \\
\left(-45^{\circ} \text { to } 45^{\circ}\right)\end{array}$ \\
\hline \multirow{3}{*}{$\mathbf{i}$} & Min/Max pow [dBm] & $-93 /-67$ & $-90 /-69$ & $-90 /-70$ \\
\hline & pow Variation $\triangle[d B]$ & 26 & 21 & 20 \\
\hline & Min/Max PL [dB] & $52 / 78$ & $54 / 75$ & $55 / 75$ \\
\hline \multirow{3}{*}{ ii } & Min/Max pow [dBm] & $-93 /-64$ & $-93 /-65$ & $-88 /-70$ \\
\hline & pow Variation $\triangle[d B]$ & 29 & 21 & 18 \\
\hline & Min/Max PL [dB] & $49 / 78$ & $50 / 78$ & $55 / 73$ \\
\hline \multirow{3}{*}{ iii } & Min/Max pow [dBm] & $-86 /-63$ & $-85 /-63$ & $-80 /-63$ \\
\hline & pow Variation $\triangle[d B]$ & 23 & 22 & 17 \\
\hline & $\operatorname{Min} / \operatorname{Max} P L[d B]$ & $48 / 71$ & $48 / 70$ & $48 / 65$ \\
\hline
\end{tabular}

Table III summarizes the measured PL for the three positions of the receiver inside the car. As expected, the worst position is scenario (i) - the pocket in the driver's door because it's more confined between metal parts and the driver's body. Table IV shows the measured car body effect as defined by (2) and described in section III. The calibration is carried out only for the case in which the transmitter is positioned at $90^{\circ}$, because it corresponds to the point where $\mathrm{P}_{\mathrm{RX}}$ is at maximum, for all the cases since the transmitter is pointed toward the receiver. To be able to evaluate the car body effect for different receiver's positions, the calibration was repeated for all three different setups (i-iii).

TABLE IV. MEASURED CAR BODY EFFECT FOR $90^{\circ}$ ANGLE.

\begin{tabular}{|c|c|c|c|c|c|}
\hline \multirow{2}{*}{ Frequency } & \multirow{2}{*}{ Setup } & \multicolumn{4}{|c|}{ Car body attenuation [dB] } \\
\cline { 3 - 6 } & & $\boldsymbol{F L}$ & $\boldsymbol{F R}$ & $\boldsymbol{R L}$ & $\boldsymbol{R} \boldsymbol{R}$ \\
\hline \multirow{3}{*}{$\mathbf{4 3 4 M H z}$} & $\boldsymbol{i}$ & 12 & 12 & 5 & 7.5 \\
\cline { 2 - 6 } & $\boldsymbol{i i}$ & 0 & 15.5 & 3 & 15 \\
\cline { 2 - 6 } & $\boldsymbol{i i i}$ & 10.5 & 14 & 15.5 & 12.5 \\
\hline $\mathbf{2 . 4 G H z}$ & $\boldsymbol{i i}$ & 6.6 & 18.4 & 17.9 & 13.3 \\
\hline
\end{tabular}

The reported values for car body effects include the driver's body effect with a person on the driver's seat to mimic the real life circumstances. This is the reason why the car body effect does not appear totally symmetrical in the measurements. Table IV shows that the contribution of car body effect in PL at $434 \mathrm{MHz}$ is between $0 \mathrm{~dB}$ and $16 \mathrm{~dB}$, which is a quite a low attenuation compared with total PL. It can be explained by the low attenuation provided by the windows and coherent addition of multiple reflected paths. The latter does not allow to apply a scalable model of the attenuation over frequency and distance.

\section{B. Measurement Results at $2.4 \mathrm{GHz}$}

The PL characterization at $2.4 \mathrm{GHz}$ has been done only for the setup (ii) - central console - as indicated in Fig. 2. The four curves are corresponding to the wheels of the car and the acronyms are the same as for the $434 \mathrm{MHz}$ measurements. The transmitted power is $2.5 \mathrm{dBm}$ and each antenna loss is estimated around $4 \mathrm{~dB}$, therefore PL can be read by adding 5.5 $\mathrm{dB}$ to the power level of Fig. 6 . To have a better understanding, the extreme limits of the estimated PL are summarized in Table $\mathrm{V}$.

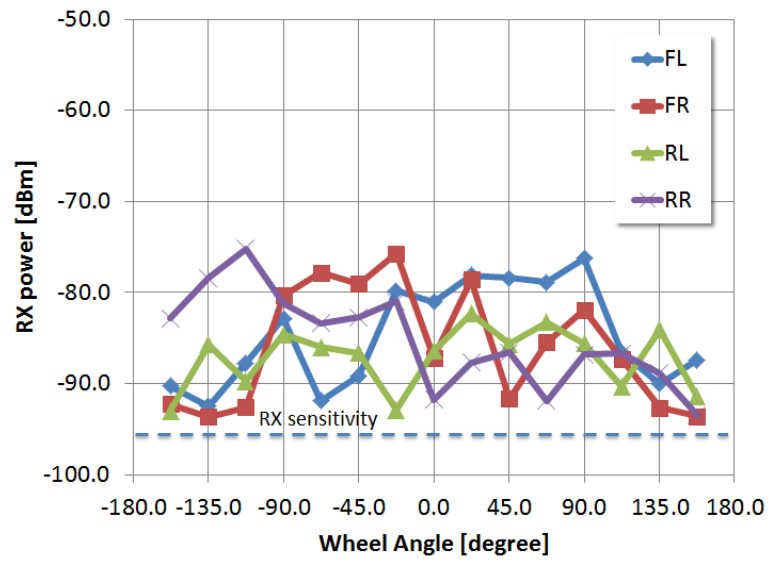

Fig. 6. Received power at $2.4 \mathrm{GHz}$ for the $\mathrm{RX}$ antenna positioned in the setup (ii). $\mathrm{P}_{\mathrm{TX}}=2.5 \mathrm{dBm}$ and estimated antennas losses are $8 \mathrm{~dB}$.

TABLE V. RX POWER OVER DIFFERENT ANGLE RANGE (ZIGBEE ON CENTRAL CONSOLE).

\begin{tabular}{|c|c|c|c|}
\hline & Full angle & $\begin{array}{c}\text { Top half } \\
\left(-90^{\circ} \text { to } 90^{\circ}\right)\end{array}$ & $\begin{array}{l}\text { Top quarter } \\
\left(-45^{\circ} \text { to } 45^{\circ}\right)\end{array}$ \\
\hline Min/Max pow [dBm] & $-93 /-75$ & $-92 /-76$ & $-92 /-76$ \\
\hline pow Variation $\triangle[d B]$ & 18 & 16 & 16 \\
\hline Min/Max PL [dB] & $69.5 / 87.5$ & $70.5 / 88.5$ & $70.5 / 88.5$ \\
\hline
\end{tabular}

Fig. 6 shows that, unlike the case of $434 \mathrm{MHz}$, restricting the operating angle does not help to increase the power level and reduce power variations; indeed minimum peaks are distributed over all the entire wheel angles. Moreover, the power difference between front and rear wheels is not evident in this case; instead a correlation with the antenna pointing seems to be dominant. An average PL of $15 \mathrm{~dB}$ higher than in the $434 \mathrm{MHz}$ case is noticed. Table IV shows that the maximum contribution of car-body-effect in the PL is less than $18.5 \mathrm{~dB}$.

\section{CHANNEl CHARACTERIZATION FOR UWB BAND}

We have investigated the feasibility of an Ultra-Wideband Impulse Radio (UWB-IR) communication because this technology offers the lowest power consumption at the transmitter side; therefore it's particularly suitable for energy harvesting powered system like sensors for smart tires. The characterization of the UWB communication is more qualitative than quantitative due to the limited measurement capabilities of the used system. Nevertheless, to the authors' best knowledge, it is the first time that this technology is tested for the communication between smart tires and the vehicle's interior. The devices used for the characterization are a pair of UWB-IR transmitter and receiver developed in our lab for indoor positioning. The system works in the bandwidth between $3.4 \mathrm{GHz}$ and $4.8 \mathrm{GHz}$. The transmitter is capable to send a 6-pulse long identification sequence (ID) at -51 $\mathrm{dBm} / \mathrm{MHz}$ effective radiated power spectral density and consuming only $85 \mathrm{uW}$ [10]. The receiver consists of a RF down-converter followed by an 8 bit $-2.88 \mathrm{Gs} / \mathrm{s}$ ADC and a software defined radio implemented in a field programmable gate array (FPGA). It shows a sensitivity of about $-80 \mathrm{dBm}$ [11]. Since the received power level is not provided by the 
system, a PL characterization was not possible, but a qualitative estimation of the link performances for the setup (iii) has been done for five angular positions on top part of each wheel $\left(-90^{\circ},-45^{\circ}, 0^{\circ}, 45^{\circ}\right.$ and $\left.90^{\circ}\right)$. Link performances have been evaluated counting the number of ID packets that were successfully received. Fig. 7 shows that received packet rate is above $80 \%$ for all the wheels when the transmitter is located on top of the wheel and at $45^{\circ}$ looking toward the receiver. However, for some cases such as $-90^{\circ}$ or $-45^{\circ}$ FL no packet was received due to the attenuation level and limited sensitivity of the receiver. As expected, the UWB link experiences higher attenuation and directivity than the lower frequencies counterparts.

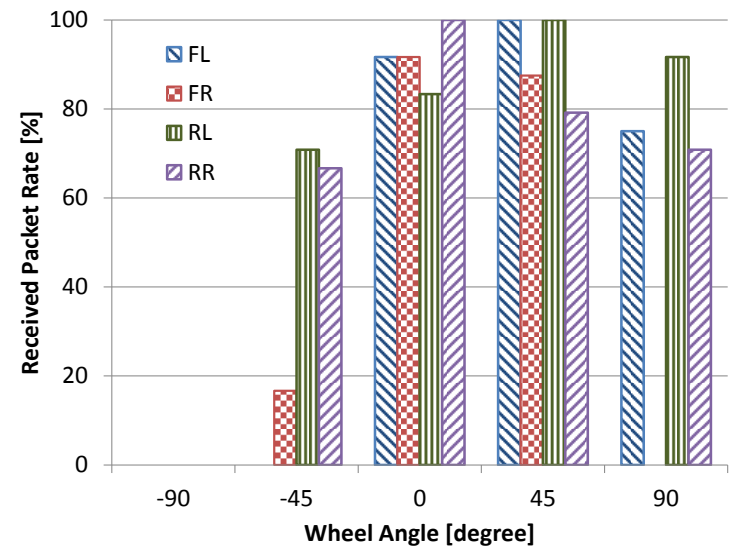

Fig. 7. Received ID packets rate for UWB-IR system in setup (iii).

\section{CONCLUSION}

In this paper the characterization of the PL between the surface of car tires and a receiver inside the car are presented for three frequencies: $434 \mathrm{MHz}$ as used by the European RKE standard, $2.4 \mathrm{GHz}$ as used by BT low-energy, and $4.1 \mathrm{GHz}$ as used by the UWB radio. This characterization is useful to analyze the power budget for wireless autonomous-powered sensors in the case of smart tire sensors embedded outside the tire's metal belt. The measurement setup was designed in a way to simulate the real life circumstance. The receiver's places in the car have been chosen in regard of the actual position of the RKE and BT receivers inside the car. The transmitter was rotated around the wheel, in step of $22.5^{\circ}$, to simulate car movements. The calibration procedure, in absence of car, was employed to break down the PL and show the contribution of the car-bodyeffects. It shows a PL higher than the FSPL model prediction and variations of around $20 \mathrm{~dB}$ due to only ground effect and antenna patterns. Measurement results at $434 \mathrm{MHz}$ show a PL between 48 and $78 \mathrm{~dB}$ depending on the positions of the transmitter and receiver. Attenuation peaks are noted and not only when the transmitting antenna is on the bottom position. Indeed, $18 \mathrm{~dB}$ of variation can be attributed to the transmitting antenna pattern. It can be observed that the position of the receiver antenna in setup (iii) is better than the other setups because the receiver antenna is less confined inside the car metal parts. Moreover, restricting the operational angle to the top quarter of the wheel reduces the variation of about 6-10 dB. At $2.4 \mathrm{GHz}$, the usual BT receiver position - setup (ii) - was employed. A PL of $15 \mathrm{~dB}$ higher than in the $434 \mathrm{MHz}$ case was noticed and power differences between front and rear wheels were not evident in this case. Using calibration procedure a carbody-effect of less than $16 \mathrm{~dB}$ and $18.5 \mathrm{~dB}$ has been estimated for $434 \mathrm{MHz}$ and $2.4 \mathrm{GHz}$, respectively. It indicates that car body attenuation does not play a big role in the PL in comparison to ground effects and antenna gain. The latter can be subject to improvement. Finally, the feasibility of employing the UWB band for communication between smart tires and car interior has been investigated, motivated by the extremely low power consumption of UWB-IR transmitters. The results of a qualitative estimation of the link performances, for the setup (iii), show a packet rate above $80 \%$ for all the wheels when the transmitter is at $0^{\circ}$ and $45^{\circ}$. This indicates that the UWB link experiences higher attenuation and directivity than the lower frequencies counterparts.

\section{REFERENCES}

[1] US Department of Transportation National Highway Traffic Safety Administration, "TIRE PRESSURE MONITORING SYSTEM FMVSS No. 138," 2005. [Online]. Available: http://www.nhtsa.gov/. [Accessed: 01-Oct-2013].

[2] R. Matsuzaki and A. Todoroki, "Wireless Monitoring of Automobile Tires for Intelligent Tires," Sensors, vol. 8, no. 12, pp. 8123-8138, Dec. 2008 .

[3] B. Mishra, C. Botteron, G. Tasselli, C. Robert, P. a. Farine, P. Janphuang, D. Briand, and N. F. de Rooij, "A sub 100 $\mu$ W UWB sensornode powered by a piezoelectric vibration harvester," in 2012 IEEE International Conference on Wireless Information Technology and Systems (ICWITS), pp. 1-4, 2012

[4] S. C. Ergen, A. Sangiovanni-Vincentelli, R. Tebano, S. Alalusi, G. Audisio, and M. Sabatini, "The Tire as an Intelligent Sensor," IEEE Trans. Comput. Des. Integr. Circuits Syst., vol. 28, no. 7, pp. 941-955, Jul. 2009.

[5] M. Cheikh, J.-G. Tartarin, J. David, S. Kessler, and A. Morin, "Transmission quality evaluation of Tire Pressure Monitoring Systems," in 2009 12th International IEEE Conference on Intelligent Transportation Systems, pp. 1-5, 2009.

[6] C. U. Bas and S. C. Ergen, "Ultra-wideband Channel Model for Intravehicular Wireless Sensor Networks Beneath the Chassis: From Statistical Model to Simulations," IEEE Trans. Veh. Technol., vol. 62, no. 1, pp. 14-25, Jan. 2013.

[7] Silicon Laboratories, "AN369 - Si4010 ANTENNA INTERFACE AND MATCHING NETWORK GUIDE," 2011. [Online]. Available: http://www.silabs.com/. [Accessed: 03-Oct-2013].

[8] Taoglas antenna solutions, "433MHz ISM Band Terminal Antenna Omni-directional, with a peak gain of 0dBi, TI.10.0112." [Online]. Available: http://www.taoglas.com/. [Accessed: 01-Oct-2013].

[9] NXP Laboratories, "Data Sheet: JN5148-001-Myy JenNet, ZigBee PRO and IEEE802.15.4 Module," 2010. [Online]. Available: http://www.jennic.com/. [Accessed: 01-Oct-2013].

[10] C. Robert, P. Tome, C. Botteron, P.-A. Farine, R. Merz, and A. Blatter, "Low power ASIC transmitter for UWB-IR radio communication and positioning," in 2010 International Conference on Indoor Positioning and Indoor Navigation, 2010, vol. 2, pp. 1-7, September.

[11] P. Tome, C. Robert, R. Merz, C. Botteron, A. Blatter, and P.-A. Farine, "UWB-based Local Positioning System: From a small-scale experimental platform to a large-scale deployable system," in 2010 International Conference on Indoor Positioning and Indoor Navigation, pp. 1-10, 2010. 